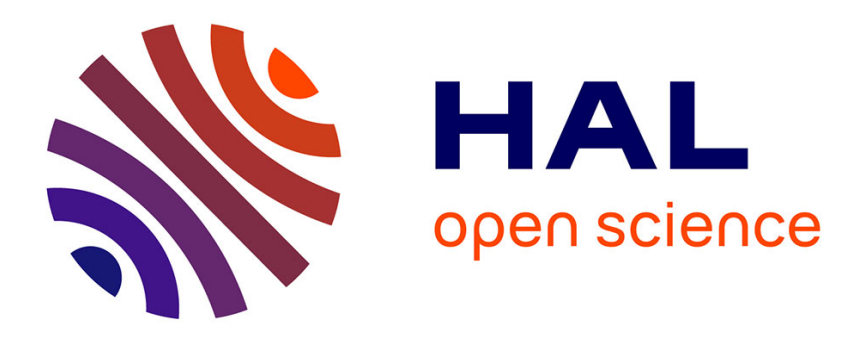

\title{
New Clustering methods for interval data
}

Marie Chavent, Francisco de A.T. de Carvahlo, Yves Lechevallier, Rosanna

\section{Verde}

\section{To cite this version:}

Marie Chavent, Francisco de A.T. de Carvahlo, Yves Lechevallier, Rosanna Verde. New Clustering methods for interval data. Computational Statistics, 2006, 21, pp.211-229. 10.1007/s00180-006-02600. hal-00260959

\section{HAL Id: hal-00260959 \\ https://hal.science/hal-00260959}

Submitted on 5 Mar 2008

HAL is a multi-disciplinary open access archive for the deposit and dissemination of scientific research documents, whether they are published or not. The documents may come from teaching and research institutions in France or abroad, or from public or private research centers.
L'archive ouverte pluridisciplinaire HAL, est destinée au dépôt et à la diffusion de documents scientifiques de niveau recherche, publiés ou non, émanant des établissements d'enseignement et de recherche français ou étrangers, des laboratoires publics ou privés. 


\title{
New clustering methods for interval data
}

\author{
Marie Chavent, Francisco de A.T. de Carvalho, Yves \\ Lechevallier and Rosanna Verde
}

MAB-Mathématiques Appliquées de Bordeaux, Université Bordeaux1, 351 cours de la libération, 33405 Talence cedex, France

CIn - Centro de Informática UFPE - Universidade Federal de Pernambuco Av. Prof. Luiz Freire s/n - Cidade Universitária CEP 50740-540 Recife-PE Brasil

INRIA - Institut National de Recherche en Informatique et en Automatique, Domaine de Voluceau - Rocquencourt B.P. 105 78153 Le Chesnay Cedex, France Dip. Strategie Aziendali e Metodologie Quantitative - SUN Seconda Università di Napoli, Corso Gran Priorato di Malta, 81043 Capua, Italie

\section{Summary}

In this paper we propose two clustering methods for interval data based on the dynamic cluster algorithm. These methods use different homogeneity criteria as well as different kinds of cluster representations (prototypes). Some tools to interpret the final partitions are also introduced. An application of one of the methods concludes the paper.

Computational Statistics 21, 211-229. 2006. 
Keywords: Dynamic clustering, interval data, distances, prototypes 


\section{Introduction}

In this paper we propose two new methods suitable for clustering a special kind of data, called symbolic data, and characterized by multi-valued descriptors (Diday (1988), Bock \& Diday (2000)). Such data are usually collected in a symbolic data table, where the individuals are represented in the rows and the multi-valued variables in the columns. The cells contain multi-values (intervals, multi-categories, distributions). In the present context of interval data analysis, each cell contains an interval of real values. In Table 1, we show an example of an interval data table, where the columns represent the monthly intervals of temperatures and the rows (objects) describe 60 Chinese meteorological stations.

\begin{tabular}{|l|c|c|c|c|c|}
\hline Stations & January & February & $\ldots$ & November & December \\
\hline \hline AnQing & {$[1,8: 7,1]$} & {$[2,1: 7,2]$} & $\ldots$ & {$[7,8: 17,9]$} & {$[4,3: 11,8]$} \\
\hline BaoDing & {$[-7,1: 1,7]$} & {$[-5,3: 4,8]$} & $\ldots$ & {$[0,8: 14]$} & {$[-3,9: 5,2]$} \\
\hline BeiJing & {$[-7,2: 2,1]$} & {$[-5,9: 3,8]$} & $\ldots$ & {$[1,5: 12,7]$} & {$[-4,4: 4,7]$} \\
\hline$\ldots$ & $\ldots$ & $\ldots$ & $\ldots$ & $\ldots$ & $\ldots$ \\
\hline ZhiJiang & {$[2,7: 8,4]$} & {$[2,7: 8,7]$} & $\ldots$ & {$[8,2: 20]$} & {$[5,1: 13,3]$} \\
\hline
\end{tabular}

Table 1: Monthly averages of minimal and maximal daily temperatures observed on 60 Chinese meteorological stations in 1988

Symbolic Data Analysis has provided partitioning methods in which different types of symbolic data are considered. Diday \& Brito (1989) used a transfer algorithm to partition a set of symbolic objects into clusters described by distribution vectors. Ralambondrainy (1995) extended the classical $k$-means clustering method in order to deal with data characterized by numerical and categorical variables. Verde et al. (2000) generalized the dynamic clustering algorithm to partition categorical multivalued symbolic data. In this approach a context-dependent proximity measure is used as an allocation function. Gordon (2000) presented an iterative relocation algorithm to partition a set of symbolic objects into classes so as to minimize the sum of the description potentials of the classes. Bock (2001) proposed several clustering algorithms for symbolic data described by interval variables, based on a clustering criterion and thereby generalized similar approaches in classical data analysis. Recently, De Souza \& De Carvalho (2004) have proposed partitioning clustering methods for interval data based on city-block distances.

The two new clustering approaches presented in this paper are based on the Dynamic Cluster Algorithm (DCA) introduced by Diday (1971), Diday \& Simon (1976). Recall that DCA needs to define an allocation function (a dissimilarity measure) and a way to represent the classes (prototypes). The methods hereafter proposed are defined with different dissimilarity measures, criteria and prototypes. 
In the first approach the prototypes are defined in the same representation space as the objects to be clustered. Hence, the prototypes are described by vectors of intervals. The dissimilarity function used to compare an object to the description of a prototype (more generally, to compare two vectors of intervals) is based on the Hausdorff distance (Chavent \& Lechevallier (2002)). In the second approach, the prototypes and the objects are not in the same description space and the comparison function is not a dissimilarity but a matching function.

Some criteria are proposed to interpret the quality of the partitions achieved by the first or the second clustering method. An application of the first method is performed on real data: 60 meteorological Chinese stations described each month by an interval of temperatures (Long-Term Instrumental Climatic Data Base of the People's Republic of China http://dss.ucar.edu/ datasets/ds578.5/data/).

\section{Notations and definitions}

An interval variable $Y$ is a correspondence from a set $E$ into the set of real values $\Re$ which has the following property on its graph: for all $s \in E$, the sub-set $[a, b]=Y(s)$ is a bounded interval of $\Re$.

Let $E=\{1, \ldots, s, \ldots, n\}$ be a set of $n$ objects described by $p$ interval variables $Y_{1}, \ldots, Y_{j}, \ldots, Y_{p}$. The interval data table is then a matrix $\left(x_{s}^{j}\right)_{n \times p}$ where the $n$ rows describe the $n$ objects to be clustered and the $p$ columns correspond to the $p$ interval variables. Each cell of the data table contains a bounded interval $x_{s}^{j}=\left[a_{s}^{j}, b_{s}^{j}\right]$ of $\Re$.

We will note :

- $x_{s}=\left(x_{s}^{1}, \ldots, x_{s}^{p}\right)$ the vector of intervals describing the object $s$

- $P=\left(C_{1}, \ldots, C_{i}, \ldots, C_{k}\right)$ a partition in $k$ clusters of $E$

- $G_{i}=\left(g_{i}^{1}, \ldots, g_{i}^{j}, \ldots, g_{i}^{p}\right)$ a prototype of the cluster $C_{i}$

- $\Lambda$ a representation space of the prototype $G_{i}$

\section{The dynamic cluster algorithm}

The aim of the dynamic cluster algorithm is to find a partition $P^{*}=\left(C_{1}, \ldots, C_{k}\right)$ of $E$ in $k$ non empty clusters and a vector $L^{*}=\left(G_{1}, \ldots, G_{i}, \ldots, G_{k}\right)$ of $k$ prototypes so that both $P^{*}$ and $L^{*}$ optimize a criterion $\Delta$ : 


$$
\Delta\left(P^{*}, L^{*}\right)=\operatorname{Min}\left\{\Delta(P, L) / P \in P_{k}, L \in \Lambda^{k}\right\}
$$

with $P_{k}$ the set of all the $k$-clusters partitions of $E$ and $\Lambda$ the representation space of the prototypes .

The criterion $\Delta$ measures the adequacy between a partition $P$ and a vector $L$ of $k$ prototypes. This criterion is defined as the sum on the $k$ clusters $C_{i}$ and on all the objects $s \in C_{i}$ of dissimilarities $D\left(x_{s}, G_{i}\right)$ :

$$
\Delta(P, L)=\sum_{i=1}^{k} \sum_{s \in C_{i}} D\left(x_{s}, G_{i}\right)
$$

This algorithm alternately performs a representation and an allocation step. In the particular case of classical (non interval) real data and of prototypes defined as the barycenters of the clusters, the dynamic cluster algorithm is equivalent to the $k$-means batch algorithm.

In order to introduce the next sections, we recall the general scheme of the DCA:

\section{DYNAMIC CLUSTER ALGORITHM (DCA)}

a) Initialization: Start from a random partition $P=\left(C_{1}, \ldots, C_{i}, \ldots, C_{k}\right)$ or, alternatively, from a vector $\left(G_{1}, \ldots, G_{i}, \ldots, G_{k}\right)$ of $k$ prototypes randomly chosen among the elements of $E$. In such a case, an allocation step is achieved as follows:

- $C_{i}=\emptyset$ for $i=1, \ldots, k$

- For $s=1$ to $n$ do:

* Assign $s$ to cluster $C_{l}$ such that $l=\operatorname{argmin}_{i=1, \ldots, k} D\left(x_{s}, G_{i}\right)$

* $C_{l}=C_{l} \cup\{s\}$

b) representation step: for $i=1$ to $k$, perform the prototype $G_{i}$ which minimizes the criterion:

$$
f_{C_{i}}(G)=\sum_{s \in C_{i}} D\left(x_{s}, G\right), \quad G \in \Lambda
$$

c) allocation step

- test $\longleftarrow 0$

- for $s=1$ to $n$ do:

* Find the cluster $C_{m}$ to which $s$ belongs 
* Find the index $l$ such that:

$$
l=\operatorname{argmin}_{i=1, \ldots, k} D\left(x_{s}, G_{i}\right)
$$

$*$ if $l \neq m$

- test $\longleftarrow 1$

- $C_{l}=C_{l} \cup\{s\}$ and $C_{m}=C_{m}-\{s\}$

d) if test $=0$ then stop, otherwise go to b)

At each iteration of this algorithm, a new couple $(P, L)$ is found and the decrease of the $\Delta$ criterion can be proved under the following conditions:

- uniqueness of the affectation cluster for each object $s \in E$

- uniqueness of the prototype $G_{i}$ minimizing the criterion $f_{C_{i}}$ given in (3) for all the clusters $C_{i}$ of the partition $P$ of $E$.

The uniqueness of a cluster to which an object $s$ is allocated (in the case of equality of the distances) is easy to solve by assigning $s$ to the cluster having the smallest index.

The existence and the uniqueness of the prototype $G_{i}$ is however more difficult to prove because it depends on the comparison function $D$. In the next section we propose two different prototypes for a cluster of interval data, related to the choice of two different comparison functions $D$ which are then parameters of two different clustering algorithms.

\section{Two new clustering methods}

The prototype $G$ of a cluster $C$ is defined according to the criterion $f_{C}(G)$ (defined in (3)) and optimized as an adequacy measure between the prototype and the cluster. Because this criterion is based on the function $D$, chosen to compare the prototype and an object to be clustered, two prototypes will be defined according to the choice of the two different comparison functions $D$.

\subsection{The first method}

The first dynamical clustering method, here proposed, compares two vectors of intervals $x_{1}$ and $x_{2}$ with a distance $d_{1}$ based on the Hausdorff distance. We do not use here the Hausdorff distance on a real $\Re^{p}$-set, as in Chavent (2004), but the sum of Hausdorff distances between intervals. First, we recall the definition of the Hausdorff distance in the case of two intervals and, 
starting from this definition, we deduce the distance $d_{1}$ between two vectors of intervals used in this first approach. Then, we look for an explicit formula of the prototype $G$ of the cluster $C$ able to optimize the adequacy criterion $f_{C}$ (Chavent \& Lechevallier (2002)), based on $d_{1}$.

\subsubsection{Definition of the Hausdorff-based distance}

The Hausdorff distance (Nadler 1978), (Rote 1991) is often used in image processing (Huttenlocher et al. 1993). It is used to compare two sets of objects $A$ and $B$. Such a distance $d_{H}$ depends on the particular metric $d\left(L_{1}\right.$ norm, $L_{2}$ norm, etc.) chosen to compare two objects $u$ and $v$ in $A$ and $B$, respectively:

$$
d_{H}(A, B)=\max (h(A, B), h(B, A))
$$

where

$$
h(A, B)=\sup _{u \in A} \inf _{v \in B} d(u, v)
$$

Let $A$ and $B$ be two intervals in $\Re, d(u, v)$ is simply $|u-v|$ and it is easy to show that the Hausdorff distance between two intervals $x_{1}^{j}=\left[a_{1}^{j}, b_{1}^{j}\right]$ and $x_{2}^{j}=\left[a_{2}^{j}, b_{2}^{j}\right]$ is:

$$
d_{H}\left(x_{1}^{j}, x_{2}^{j}\right)=\max \left(\left|a_{1}^{j}-a_{2}^{j}\right|,\left|b_{1}^{j}-b_{2}^{j}\right|\right)
$$

Finally, the distance $d_{1}$ between two vectors of intervals $x_{1}$ and $x_{2}$ is the sum on the $p$ variables of the Hausdorff distances between the intervals:

$$
d_{1}\left(x_{1}, x_{2}\right)=\sum_{j=1}^{p} \max \left(\left|a_{1}^{j}-a_{2}^{j}\right|,\left|b_{1}^{j}-b_{2}^{j}\right|\right)
$$

For intervals reduced to single points, that is when $a^{j}=b^{j}, d_{1}$ is the $L_{1}$ distance in $\Re^{p}$.

\subsubsection{The prototype}

The prototype $G=\left(g^{1}, \ldots, g^{p}\right)$ of a cluster $C$ is the vector of $p$ intervals which minimizes the adequacy criterion:

$$
f_{C}(G)=\sum_{s \in C} d_{1}\left(x_{s}, G\right)=\sum_{s \in C} \sum_{j=1}^{p} d_{H}\left(x_{s}^{j}, g^{j}\right)
$$

Criterion (8) can also be written as:

$$
f_{C}(G)=\sum_{j=1}^{p} \overbrace{\sum_{s \in C} d_{H}\left(x_{s}^{j}, g^{j}\right)}^{\tilde{f_{C}}\left(g^{j}\right)}
$$


and the problem is now to find the interval $g^{j}=\left[\alpha^{j}, \beta^{j}\right]$ for $(j=1, \ldots, p)$ which minimizes:

$$
\tilde{f_{C}}\left(g^{j}\right)=\sum_{s \in C} d_{H}\left(x_{s}^{j}, g^{j}\right)=\sum_{s \in C} \max \left(\left|\alpha^{j}-a_{s}^{j}\right|,\left|\beta^{j}-b_{s}^{j}\right|\right)
$$

We will see how to solve this minimization problem by transforming it into two well-known minimization problems. Let $m_{s}^{j}$ be the midpoint of an interval $x_{s}^{j}=\left[a_{s}^{j}, b_{s}^{j}\right]$ and $l_{s}^{j}$ be half of its length, i.e.:

$$
m_{s}^{j}=\frac{a_{s}^{j}+b_{s}^{j}}{2} \text { and } l_{s}^{j}=\frac{b_{s}^{j}-a_{s}^{j}}{2}
$$

and let $\mu^{j}$ and $\lambda^{j}$ be the midpoint and half-length of the interval $g^{j}=\left[\alpha^{j}, \beta^{j}\right]$, respectively. According to the following property defined for $x$ and $y$ in $\Re$ :

$$
\max (|x-y|,|x+y|)=|x|+|y|
$$

the function (10) can be written as:

$$
\begin{aligned}
\tilde{f_{C}}\left(g^{j}\right) & =\sum_{s \in C} \max \left(\left|\left(\mu^{j}-\lambda^{j}\right)-\left(m_{s}^{j}-l_{s}^{j}\right)\right|,\left|\left(\mu^{j}+\lambda^{j}\right)-\left(m_{s}^{j}+l_{s}^{j}\right)\right|\right) \\
& =\sum_{s \in C}\left|\mu^{j}-m_{s}^{j}\right|+\sum_{s \in C}\left|\lambda^{j}-l_{s}^{j}\right|
\end{aligned}
$$

This yields two well-known minimization problems: find $\mu^{j} \in \Re$ and $\lambda^{j} \in \Re$ which minimizes, respectively:

$$
\sum_{s \in C}\left|\mu^{j}-m_{s}^{j}\right| \text { and } \sum_{s \in C}\left|\lambda^{j}-l_{s}^{j}\right|
$$

The solutions $\hat{\mu^{j}}$ and $\hat{\lambda^{j}}$ are respectively the median of $\left\{m_{s}^{j}, s \in C\right\}$, which are the midpoints of the intervals $x_{s}^{j}=\left[a_{s}^{j}, b_{s}^{j}\right], s \in C$, and the median of the set $\left\{l_{s}^{j}, s \in C\right\}$ of their half-lengths. Finally, the solution $\hat{g^{j}}=\left[\hat{\alpha^{j}}, \hat{\beta^{j}}\right]$ is the interval $\left[\hat{\mu^{j}}-\hat{\lambda^{j}}, \hat{\mu^{j}}+\hat{\lambda^{j}}\right]$ and the prototype of $C$ is $G=\left(\hat{g}^{1}, \ldots, \hat{g}^{p}\right)$.

\subsection{The second method}

The second dynamic clustering method, proposed in this paper, compares two vectors of intervals by means of a dissimilarity $d_{2}$. This dissimilarity compares two couples $p_{1}^{j}=\left(S_{1}^{j}, q_{1}\right)$ and $p_{2}^{j}=\left(S_{2}^{j}, q_{2}\right)$, where $q_{1}$ and $q_{2}$ are weight functions associated, respectively, to suitable sets of intervals $S_{1}^{j}$ and $S_{2}^{j}$. 
These subsets are obtained by a pre-processing step descretizing the intervals $x_{1}^{j}$ and $x_{2}^{j}$ in "basic" or elementary intervals. Firstly, we describe how to get such subsets of intervals and the associating weight functions. Then, we introduce the "two components" dissimilarity measure $d_{2 c}$ to compare $p_{1}^{j}$ and $p_{2}^{j}$ and we define $d_{2}$ as the sum of those dissimilarities with respect to the $p$ variables. Finally, we perform a prototype $G$.

\subsubsection{Pre-processing step}

The aim of the pre-processing step (De Carvalho (1995), De Carvalho et al. (1999), Chavent et al. (2003)) is to discretize $x_{s}^{j}$ to obtain a subset of a set $\left\{I_{1}^{j}, \ldots, I_{H_{j}}^{j}\right\}$ of elementary intervals and the corresponding set of weights $q_{s}^{j}$.

A column $j$ of the data table is a set $\left\{x_{1}^{j}, \ldots, x_{s}^{j}, \ldots, x_{n}^{j}\right\}$ of $n$ intervals. Starting from this set of intervals, another set of $H_{j}$ disjoint intervals $\left\{I_{1}^{j}, \ldots, I_{h}^{j}, \ldots, I_{H_{j}}^{j}\right\}$ is performed. The elementary intervals are obtained by sorting the set of lower and upper bounds of the $n$ intervals $\left\{x_{1}^{j}, \ldots, x_{s}^{j}, \ldots, x_{n}^{j}\right\}$. The so called "elementary" intervals $I_{h}^{j}$ have to verify the following properties:

i) $\bigcup_{h=1}^{H_{j}} I_{h}^{j}=\bigcup_{s=1}^{n} x_{s}^{j}$

ii) $I_{h}^{j} \cap I_{h^{\prime}}^{j}=\emptyset$ if $h \neq h^{\prime}$

iii) $\forall s \in E, \forall h \quad I_{h}^{j} \subseteq x_{s}^{j}$ or $I_{h}^{j} \cap x_{s}^{j}=\emptyset$

iv) $\forall s \in E, \exists S_{s}^{j} \subset\left\{I_{1}^{j}, \ldots, I_{H_{j}}^{j}\right\}: \bigcup_{I_{h}^{j} \in S_{s}^{j}} I_{h}^{j}=x_{s}^{j}$ and $\forall I_{h}^{j} \in S_{s}^{j}, I_{h}^{j} \subseteq x_{s}^{j}$

Then, $S_{s}^{j}=\left\{I_{h}^{j}: I_{h}^{j} \subseteq x_{s}^{j}\right\}$. The weight function $q_{s}$ defined on the subset of elementary intervals $S_{s}^{j}$ which discretizes the interval $x_{s}^{j}$ is then defined as:

$$
\begin{aligned}
& q_{s}: S_{s}^{j} \quad \longrightarrow \quad[0,1] \\
& I_{h}^{j} \in S_{s}^{j} \longrightarrow q_{s}\left(I_{h}^{j}\right)=\frac{\left|I_{h}^{j}\right|}{b_{s}^{j}-a_{s}^{j}}
\end{aligned}
$$

where $\left|I_{h}^{j}\right|$ is the length of the interval $I_{h}^{j}$. Notice that, $\forall I_{h}^{j} \in S_{s}^{j}, q_{s}\left(I_{h}^{j}\right) \geq 0$ and that $\sum_{I_{h}^{j} \in S_{s}^{j}} q_{s}\left(I_{h}^{j}\right)=1$.

An example is given in Figure 1. Let us consider four intervals $x_{1}^{j}, x_{2}^{j}, x_{3}^{j}$ and $x_{4}^{j}$ (describing a set $E$ of four objects on a variable $Y_{j}$ ). The set of elementary intervals $\left\{I_{1}^{j}, I_{2}^{j}, I_{3}^{j}, I_{4}^{j}, I_{5}^{j}\right\}$ is shown and, because the set of elementary intervals which discretize the interval $x_{1}^{j}$ is $\left\{I_{1}^{j}, I_{2}^{j}\right\}$, their associated weights are $q_{1}\left(I_{1}^{j}\right)=\alpha$ and $q_{1}\left(I_{2}^{j}\right)=1-\alpha$. 
Figure 1: Elementary intervals construction

\subsubsection{Definition of the "two components" dissimilarity}

As in the first method, where we compared two intervals $x_{1}^{j}$ and $x_{2}^{j}$ (for each variable $j$ ) by the Hausdorff distance, here we compare the couples $p_{1}^{j}=\left(x_{1}^{j}, q_{1}\right)$ and $p_{2}^{j}=\left(x_{2}^{j}, q_{2}\right)$ by a "two components" dissimilarity measure, noted as $d_{2 c}$, and defined as:

$$
d_{2 c}\left(p_{1}^{j}, p_{2}^{j}\right)=d_{c i}\left(x_{1}^{j}, x_{2}^{j}\right)+d_{c d}\left(q_{1}, q_{2}\right)
$$

where $d_{c i}$ is a dissimilarity which measures the difference in "position" between two intervals, and $d_{c d}$ is a dissimilarity between two weight functions $q_{1}$ and $q_{2}$ which express the difference in content between two intervals (De Carvalho \& Souza (1998)).

The first "component" of the dissimilarity $d_{2 c}$ is the dissimilarity $d_{c i}$ between two intervals $x_{1}^{j}=\left[a_{1}^{j}, b_{1}^{j}\right]$ and $x_{2}^{j}=\left[a_{2}^{j}, b_{2}^{j}\right]$ defined by:

$$
d_{c i}\left(x_{1}^{j}, x_{2}^{j}\right)=\frac{\left|\left(\bar{x}_{1}^{j} \cap \bar{x}_{2}^{j}\right) \cap\left(x_{1}^{j} \oplus x_{2}^{j}\right)\right|}{\left|x_{1}^{j} \oplus x_{2}^{j}\right|}
$$

where:

- |.| the length of an interval

- $x_{1}^{j} \oplus x_{2}^{j}=\left[\min \left(a_{1}^{j}, a_{2}^{j}\right), \max \left(b_{1}^{j}, b_{2}^{j}\right)\right]$

- $\left.\bar{x}_{s}^{j}=\right]-\infty, a_{s}^{j}[\cup] b_{s}^{j},+\infty\left[\right.$ the complementary set of $x_{s}^{j}$ in $\Re$ 
Another formulation of this dissimilarity is:

$$
d_{c i}\left(x_{1}^{j}, x_{2}^{j}\right)= \begin{cases}\frac{\left|\min \left(b_{1}^{j}, b_{2}^{j}\right)-\max \left(a_{1}^{j}, a_{2}^{j}\right)\right|}{\max \left(b_{1}^{j}, b_{2}^{j}\right)-\min \left(a_{1}^{j}, a_{2}^{j}\right)} & \text { if } x_{1}^{j} \cap x_{2}^{j}=\emptyset \\ 0 & \text { otherwise }\end{cases}
$$

Notice that, according to the $d_{c i}$ component, there is difference in "position" between two intervals only when the intersection between them is empty.

The second "component" of $d_{2 c}$ is the dissimilarity $d_{c d}$ between the two weight functions $q_{1}$ and $q_{2}$ defined, respectively, on subsets $S_{1}^{j}$ and $S_{2}^{j}$ of elementary intervals which discretizes $x_{1}^{j}$ and $x_{2}^{j}$ :

$$
d_{c d}\left(q_{1}, q_{2}\right)=\frac{1}{2}\left(\sum_{\left\{I_{h}^{j}: I_{h}^{j} \in S_{1}^{j}, I_{h}^{j} \notin S_{2}^{j}\right\}} q_{1}\left(I_{h}^{j}\right)+\sum_{\left\{I_{h}^{j}: I_{h}^{j} \in S_{2}^{j}, I_{h}^{j} \notin S_{1}^{j}\right\}} q_{2}\left(I_{h}^{j}\right)\right)
$$

Notice that $0 \leq d_{c d} \leq 1$, with $d_{c d}=0$ if $x_{1}^{j}=x_{2}^{j}$ and $d_{c d}=1$ if $x_{1}^{j} \cap x_{2}^{j}=\emptyset$.

Finally the dissimilarity $d_{2}$ between the two couples $p_{1}$ and $p_{2}$ is the sum of the dissimilarities $d_{2 c}$ computed with respect to the $p$ variables $Y_{j}$ 's:

$$
d_{2}\left(p_{1}, p_{2}\right)=\sum_{j=1}^{p} d_{2 c}\left(p_{1}^{j}, p_{2}^{j}\right)=\sum_{j=1}^{p}\left(d_{c i}\left(x_{1}^{j}, x_{2}^{j}\right)+d_{c d}\left(q_{1}, q_{2}\right)\right)
$$

\subsubsection{The prototype}

The prototype $G$ of a cluster $C$ is now a vector where the components are $p$ couples $\left(\Gamma^{j}, q\right)$. The components of the prototype are defined in the following way:

- $\Gamma^{j}$ may be defined in two different ways (see Figure 2):

(a) $\Gamma^{j}=\left[\min _{s \in C} a_{s}^{j}, \max _{s \in C} b_{s}^{j}\right]$ is an interval "generalizing" the intervals $x_{s}^{j}=\left[a_{s}^{j}, b_{s}^{j}\right]$, for $s \in C$;

(b) $\Gamma^{j}=\left\{x_{s}^{j}: s \in C\right\}$.

In both the cases the intervals can be considered discretized, in the pre-processing step, into elementary intervals, as above described.

- $q$ is defined as $g=\left\{\begin{array}{l}\left.\frac{1}{\operatorname{card}(C)} \sum_{\left\{h, s: I_{h} \in S_{s}^{j}\right.} \text { and } s \in C\right\} \\ 0, \text { otherwise }\end{array}\right.$

Because in the definition (b) of $\Gamma^{j}$ the result is not an interval, the prototype is not, in this particular case, represented in the same description space as 
Figure 2: The two different ways to define $\Gamma$

the objects of the cluster. The dissimilarity $d_{2}$ cannot be directly used to compare an object $s$ and the prototype $G$. For this reason, we propose to replace in $d_{2}$ and, more precisely, in $d_{2 c}$ (in 16) the first component $d_{c i}$ by:

$$
d_{c i}^{*}\left(x_{s}^{j}, \Gamma^{j}\right)=\frac{\mid\left(\bar{x}_{s}^{j} \cap\left(\bigcap_{s^{\prime} \in C} \bar{x}_{s^{\prime}}^{j}\right)\right) \cap\left(x_{s}^{j} \oplus\left(\bigcup_{s^{\prime} \in C} x_{s^{\prime}}^{j}\right) \mid\right.}{\left.\mid x_{s}^{j} \oplus\left(\bigcup_{s^{\prime} \in C} x_{s^{\prime}}^{j}\right)\right) \mid}
$$

with $x_{s}^{j} \oplus\left(\bigcup_{s^{\prime} \in C} x_{s^{\prime}}^{j}\right)=\left[\min \left(a_{s}^{j}, \min _{s^{\prime} \in C} a_{s^{\prime}}^{j}\right), \max \left(b_{s}^{j}, \max _{s^{\prime} \in C} b_{s^{\prime}}^{j}\right)\right]$

The comparison function $D$ (used in the allocation step of the algorithm) between an object $s$ and the prototype $G$ is then defined by:

$$
d_{2}^{*}\left(p_{s}, G\right)=\sum_{j=1}^{p}\left(d_{c i}^{*}\left(x_{s}^{j}, \Gamma^{j}\right)+d_{c d}\left(q_{s}, q\right)\right)
$$

Remark: Because $p_{s}$ and $G$ are not in the same description space, $d_{2}^{*}$ is not a dissimilarity function but a "matching" function.

Moreover, the prototype $G$ here defined doesn't minimize the adequacy function (as in the representation step of the dynamic cluster algorithm). For this reason this second clustering approach is not a proper dynamic cluster method and an exchange algorithm is performed.

\subsubsection{The algorithm}

The algorithm used in the second method is based on the exchange algorithm (Chavent et al. (2003)). At each iteration of this algorithm the $n$ objects are considered one after the other and are assigned to the cluster such that the decrease of the $\Delta$ criterion is maximum. As in the first version of the well known k-means algorithm, the prototypes are updated at each moving of an object from one cluster to another: 


\section{EXCHANGE ALGORITHM:}

a) Initialization: Start from a random partition $P=\left(C_{1}, \ldots, C_{i}, \ldots, C_{k}\right)$

c) allocation step

- test $\longleftarrow 0$

- for $s=1$ to $n$ do:

* Find the cluster $C_{m}$ to which $s$ belongs

* If $\operatorname{card}\left(C_{m}\right) \neq 1$ for $l=1, \ldots, k$ and $l \neq m$

- perform the new prototypes $G_{m}$ of $C_{m}-\{s\}$ and $G_{l}$ of $C_{l} \cup\{s\}$

. perform the criterion $\Delta_{l}=\sum_{i=1}^{k} \sum_{s^{\prime} \in C_{i}} D\left(p_{s^{\prime}}, G_{i}\right)$ where $D$ can be $d_{2}$ or $d_{2}^{*}$ according to the selected type of prototype

* find the cluster $C_{l^{*}}$ such that

$$
l^{*}=\arg \min _{l=1, \ldots, k} \Delta_{l}
$$

$*$ if $l^{*} \neq m$ move $s$ to $C_{l^{*}}$

. test $\longleftarrow 1$

- $C_{l^{*}}=C_{l^{*}} \cup\{s\}$ and $C_{m}=C_{m}-\{s\}$

b) if test $=0$ then stop, otherwise go to b)

\section{Interpretation}

The aim of this section is to provide the user with various criteria to measure and interpret the quality of the partition or the quality of the clusters of the partition. These criteria are obtained by generalizing some criteria proposed in Celeux et al. (1989) for a partition $P$ of $n$ points $x_{s}$ of $\Re^{p}$ weighted by $p_{s}$ and performed by dynamical clustering. All these criteria are based on the decomposition of the total inertia into within-cluster and between-cluster inertia. In order to simplify our presentation we take $p_{s}=1(\forall s=1, \ldots, n)$ and, in this particular case, the inertia is the total sum of squares (TSS) of the $\Re^{p}$ points around their mean. The decomposition of the total sum of squares $(T S S)$ into the within-cluster $(W S S)$ and between-cluster $(B S S)$ sum of squares is:

$$
\underbrace{\sum_{s=1}^{n} d^{2}\left(x_{s}, G\right)}_{T S S}=\underbrace{\sum_{i=1}^{k} \sum_{s \in C_{i}} d^{2}\left(x_{s}, G_{i}\right)}_{W S S}+\underbrace{\sum_{i=1}^{k} n_{i} d^{2}\left(G_{i}, G\right)}_{B S S}
$$


where $d$ is the squared Euclidean distance, $G$ is the mean of the $n$ points $x_{s} \in E, G_{i}$ is the mean of the points $x_{s} \in C_{i}$ and $n_{i}=\operatorname{card}\left(C_{i}\right)$.

A well known result is that the mean $G$ of a cluster $C$ is the point $g \in \Re^{p}$ which minimizes the following adequacy criterion:

$$
f_{C}(g)=\sum_{s \in C} d^{2}\left(x_{s}, g\right)
$$

In the first clustering method proposed in section 4.1 we generalized the idea of the mean $G$ of a cluster $C$ to the idea of a prototype $G$ which minimizes the adequacy criterion:

$$
f_{C}(g)=\sum_{s \in C} D\left(x_{s}, g\right)
$$

where $D=d_{1}$ is based on the Hausdorff distance and the solution $G=$ $\left(\hat{g}^{1}, \ldots, \hat{g}^{p}\right)$ is a vector of intervals (defined in section 4.1.2).

The TSS (or the total inertia) and the WSS (or the within-cluster inertia) defined in (23) can then be generalized by using a prototype $G_{i}$ of a cluster $C_{i}$ which optimizes the adequacy criterion (24) for a specific comparison function $D$. We then have :

- WSS $=\sum_{i=1}^{k} \sum_{s \in C_{i}} D\left(x_{s}, G_{i}\right)=\sum_{i=1}^{k} f_{C_{i}}\left(G_{i}\right)$ which is then equal to the criterion $\Delta(P, L)$ given in (1).

- $T S S=\sum_{s=1}^{n} D\left(x_{s}, G_{E}\right)$ which is the adequacy criterion $f_{E}\left(G_{E}\right)$ defined in (24) with $G_{E}$ is the prototype of the whole set of $n$ objects in $E$.

Of course, the equality (23) is not true after generalization. The gain of inertia obtained by replacing the propotype $G$ of $E$ by the $k$ prototypes $\left(G_{1}, \ldots, G_{k}\right)$ of the partition $P$ is no more the between-cluster inertia (or $B S S$ ). The gain of homogeneity obtained by replacing the $n$ objects by the $k$ prototypes is simply defined as the difference between $f_{E}\left(G_{E}\right)$ and $\Delta(P, L)$.

Finally the three following criteria will be used to interpret a partition and its clusters:

- $f_{C_{i}}\left(G_{i}\right)$ which is a measure of homogeneity of the cluster $C_{i}$;

- $\Delta(P, L)$ which is a measure of within-cluster homogeneity of the partition $P$;

- $f_{E}\left(G_{E}\right)$ which is a measure of total homogeneity of the set $E$. 
Remark: Because in the second method the prototypes are not defined by the minimization of an adequacy criterion, the criteria given below can not be applied to interpret a partition obtained using this algorithm.

\subsection{Interpretation of the partition}

In this section we give a criterion to globally measure the quality of a $k$ clusters partition and two criteria to interpret this partition according to a variable $Y_{j}$.

The quality of a partition is measured by the gain of homogeneity obtained by replacing the $n$ objects of $E$ by the $k$ prototypes of $P$ and normalized by the total homogeneity $f_{E}\left(G_{E}\right)$ :

$$
Q(P)=1-\frac{\Delta(P, L)}{f_{E}\left(G_{E}\right)}
$$

This criterion takes its values between 0 and 1 . It is equal to 1 when all the clusters are reduced to single objects or to identical objects. It is equal to 0 for the one-cluster partition $E$. Because this criterion decreases with the number of clusters it can only be used to compare two partitions having the same number of clusters. Because a $k$-clusters partition is better than another partition in $k$ clusters if the criterion $\Delta(P, L)$ is smaller, this partition will be better if $Q(P)$ is bigger. For classical quantitative data

$$
Q(P)=\frac{B S S}{T S S}=1-\frac{W S S}{T S S}
$$

is called the part of inertia of $E$ explained by $P$. The criterion $Q(P)$ measures in the same way the part of the homogeneity of $E$ explained by $P$.

Similarly, we define the quality of the partition for each variable $Y_{j}$ as:

$$
Q_{j}(P)=1-\frac{\sum_{i=1}^{k} \tilde{f}_{C_{i}}\left(\hat{g}_{i}^{j}\right)}{\tilde{f}_{E}\left(\hat{g}_{E}^{j}\right)}
$$

which is the part of the homogeneity of the variable $Y_{j}$ explained by $P$. This criterion measures the power of discrimination of the variable $Y_{j}$ to the partition $P$. Moreover because the quality of $P, Q(P)$, is a weighted average of the values $Q_{j}(P)$ :

$$
Q(P)=\sum_{j=1}^{p} \frac{\tilde{f}_{E}\left(\hat{g}_{E}^{j}\right)}{f_{E}\left(G_{E}\right)} Q_{j}(P)
$$


this criterion also measures the importance of the variable $Y_{j}$ in the construction of the partition. Finally the criteria $Q_{j}(P)$ must be compared to $Q(P)$.

\subsection{Interpretation of the clusters}

The quality of a cluster $C_{i}$ of $E$ is defined by:

$$
Q\left(C_{i}\right)=1-\frac{f_{C_{i}}\left(G_{i}\right)}{f_{C_{i}}\left(G_{E}\right)}
$$

This criterion measures the gain of homogeneity of the cluster $C_{i}$ obtained when replacing the prototype $G_{E}$ by the prototype $G_{i}$ in the calculation of the homogeneity.

The contribution of a cluster $C_{i}$ to the within-cluster homogeneity of $P$ is defined by:

$$
K\left(C_{i}\right)=\frac{f_{C_{i}}\left(G_{i}\right)}{\Delta(P, L)}
$$

The sum of the $k$ contributions is obviously 1 .

A final criterion that is useful to interpret a cluster according to a variable $Y_{j}$ is:

$$
Q_{j}\left(C_{i}\right)=1-\frac{\tilde{f}_{C_{i}}\left(\hat{g}_{i}^{j}\right)}{\tilde{f}_{C_{i}}\left(\hat{g}_{E}^{j}\right)}
$$

This criterion measures the part of discrimination power of the variable $Y_{j}$ taken into account by $C_{i}$. In other words this criterion helps the user to find the variables which characterize the cluster $C_{i}$. Because the quality of the cluster, $Q\left(C_{i}\right)$, is a weighted average of the values $Q_{j}\left(C_{i}\right)$ :

$$
Q\left(C_{i}\right)=\sum_{j=1}^{p} \frac{\tilde{f}_{C_{i}}\left(\hat{g}_{E}^{j}\right)}{f_{C_{i}}\left(G_{E}\right)} Q_{j}\left(C_{i}\right)
$$

the values of the criterion $Q_{j}\left(C_{i}\right)$ have to be interpreted by comparison with the value $Q\left(C_{i}\right)$. In other words we will consider that a variable $Y_{j}$ characterizes the cluster $C_{i}$ if $Q_{j}\left(C_{i}\right)>Q\left(C_{i}\right)$.

\section{Application to the 60 meteorological stations in China}

The criteria proposed to interpret a partition have been applied to an interval data set extracted from the Long-Term Instrumental Climatic Database of 
the People's Republic of China. This Database contains among other variables the temperatures observed in 60 meteorological stations in China. In order to compare the 60 meteorological stations according to their temperatures, a natural representation of each station is to describe each month by an interval of minimal and maximal temperatures (see Table 1). Here we worked with the temperatures of the year 1988 and we built an interval data table of 60 rows and 12 columns corresponding to the 60 stations and the 12 months of the year.

On this data set we applied the first clustering method based on the Hausdorff distance in order to interpret the results with the criteria proposed in the previous section.

The number of clusters of the partitions was fixed to 5 . The first algorithm was repeated 50 times with different initializations and the best 5 -clusters partitions (i.e. minimizing $\Delta(P, L)$ ) were retained. We computed the quality criterion $Q(P)$ defined in $(25)$ for the best partition and we found that this partition explained $64,33 \%$ of the homogeneity.

In order to know a little bit more about the clusters of the best partition we performed their quality $Q\left(C_{i}\right)(\times 100)$ and their contribution $K\left(C_{i}\right)(\times 100)$ defined in (28) and (29) (see Table 2).

\begin{tabular}{|c|c|c|c|}
\hline$i$ & size & $Q\left(C_{i}\right)$ & $K\left(C_{i}\right)$ \\
\hline 1 & 10 & 78.57 & 13.82 \\
\hline 2 & 13 & 66.05 & 25.26 \\
\hline 3 & 17 & 47.68 & 27.99 \\
\hline 4 & 13 & 7.68 & 18.82 \\
\hline 5 & 7 & 79.11 & 14.11 \\
\hline
\end{tabular}

Table 2: Quality $(\times 100)$ and contribution $(\times 100)$ of the clusters $C_{i}$ of the best partition

Because the homogeneity of a cluster naturally increases with the number of objects, this information has to be taken into account in the interpretation of these criteria. For this reason we compare here the cluster $C_{2}$ with the cluster $C_{4}$ both of which have 13 objects. We first notice that in Table 2 $Q\left(C_{2}\right)=66,05 \%$ whereas $Q\left(C_{4}\right)=7,68 \%$. We have seen that the quality of a cluster $(\times 100)$ is the percentage of gain of homogeneity obtained by replacing the global prototype $G_{E}$ by the cluster prototype $G_{i}$. This criterion also measures the adequacy between the two prototypes $G_{E}$ and $G_{i}$. This means that here the cluster $C_{2}$ is more "atypical" than $C_{4}$ or, in other words, that the objects of $C_{4}$ are more similar to the "average" object $G_{E}$. Concerning the contributions we have $K\left(C_{2}\right)=25,26 \%$ and $Q\left(C_{4}\right)=18,82 \%$. This means that cluster $C_{4}$ is more homogeneous than $C_{1}$. 
Finally, in order to interpret the partition or the clusters according to the variables, we computed, for the best partition and for each variable $Y_{j}$, the criterion $Q_{j}(P)$ defined in (26) and, for the 5 clusters of the best partition, the criterion $Q_{j}\left(C_{i}\right)$ defined in (30) (see Table 6).

\begin{tabular}{|l|c|c|c|c|c|c|}
\hline Variable & $Q_{j}$ & $Q_{j}\left(C_{1}\right)$ & $Q_{j}\left(C_{2}\right)$ & $Q_{j}\left(C_{3}\right)$ & $Q_{j}\left(C_{4}\right)$ & $Q_{j}\left(C_{5}\right)$ \\
\hline January & $\mathbf{6 9 . 5 0}$ & $\mathbf{8 2 . 8 5}$ & $\mathbf{7 0 . 1 7}$ & $\mathbf{5 6 . 1 3}$ & $\mathbf{1 1 . 2 8}$ & $\mathbf{7 9 . 3 9}$ \\
\hline February & $\mathbf{6 6 . 1 8}$ & $\mathbf{7 7 . 6 2}$ & 63.61 & $\mathbf{4 8 . 4 3}$ & $\mathbf{8 . 4 5}$ & $\mathbf{8 2 . 8 2}$ \\
\hline March & $\mathbf{6 4 . 5 2}$ & $\mathbf{7 8 . 6 9}$ & 64.75 & 44.60 & $\mathbf{1 4 . 0 3}$ & 78.31 \\
\hline April & $\mathbf{6 4 . 3 6}$ & 72.31 & $\mathbf{7 1 . 3 9}$ & 40.78 & 6.36 & $\mathbf{8 4 . 7 5}$ \\
\hline May & 61.68 & 69.56 & $\mathbf{6 7 . 2 3}$ & 37.89 & 7.34 & $\mathbf{7 9 . 9 7}$ \\
\hline June & 53.36 & 77.41 & 63.91 & 32.88 & 5.55 & 66.87 \\
\hline July & 46.31 & 74.50 & 50.00 & 31.79 & 4.73 & 62.26 \\
\hline August & 47.19 & 75.76 & 53.99 & 28.04 & 3.24 & 46.54 \\
\hline September & 61.10 & 78.18 & 65.58 & 28.16 & 6.85 & 76.59 \\
\hline October & $\mathbf{7 0 . 4 1}$ & $\mathbf{8 2 . 9 8}$ & $\mathbf{7 5 . 0 9}$ & $\mathbf{4 9 . 5 9}$ & 7.20 & $\mathbf{8 3 . 3 7}$ \\
\hline November & $\mathbf{7 0 . 6 3}$ & $\mathbf{7 9 . 5 5}$ & $\mathbf{7 3 . 6 0}$ & $\mathbf{6 1 . 0 2}$ & 4.22 & $\mathbf{8 4 . 6 7}$ \\
\hline December & $\mathbf{7 1 . 3 3}$ & $\mathbf{8 2 . 0 1}$ & 62.55 & $\mathbf{6 8 . 3 9}$ & $\mathbf{1 1 . 2 3}$ & $\mathbf{8 1 . 8 6}$ \\
\hline \hline Threshold & 64.33 & 78.57 & 66.05 & 47.68 & 7.68 & 79.11 \\
\hline
\end{tabular}

Table 3: Quality $(\times 100)$ of each variable (month), to the best partition and its clusters $C_{i}$

Because a variable $Y_{j}$ can be considered as discriminant for a partition $P$ if $Q_{j}(P)>Q(P)$ and for the cluster $C_{i}$ if $Q_{j}\left(C_{i}\right)>Q\left(C_{i}\right)$, we have indicated in bold in Table 6 the values of $Q_{j}(p)$ and $Q_{j}\left(C_{i}\right)$ which verify these conditions. Finally we see, in Table 6 , that globally, the less discriminant variables are June, July and August i.e. the three summer months.

\section{Conclusion}

The two methods shown above can be considered as the most suitable generalization of the classical DCA to the analysis of data expressed as intervals of real values. In fact, the generalization of DCA to such a kind of data could be formalized in a different way. The proposed approaches synthesize the case of classes represented by an element belonging to the same space as the objects to be clustered, as well as the case of classes that synthesize the characteristics of the elements of a class.

As we have seen, the search for the partitions of objects described by interval variables can be achieved by an optimization process as general as for the DCA on classical data. Like for DCA in the classical context, the best fitting between the representation and the allocation function is formalized by the criterion, which is a guarantee of coherent results. Even if the proposed 
approaches appear different a priori, the results are quite similar because the model of the prototype, chosen in each approach, characterizes the same notion of coherence of a class.

In conclusion, we can remark that the choice of the kind of prototype usually guides the choice of the clustering method.

Acknowledgments: The second author would like to thank CNPq and FACEPE (Brazilian Agencies) for their financial support.

\section{References}

Bock, H.-H. (2001), Clustering algorithms and kohonen maps for symbolic data, in 'ICNCB Proceedings', Osaka, pp. 203-215.

Bock, H.-H. \& Diday, E., eds (2000), Analysis of symbolic data. Exploratory methods for extracting statistical information from complex data, Studies in classification, data analysis and knowledge organisation, Springer Verlag, Heidelberg.

Celeux, G., Diday, E., Govaert, G., Lechevallier, Y. \& Ralambondrainy, H. (1989), Classification automatique des donnes, Dunod.

Chavent, M. (2004), An hausdorff distance between hyper-rectangles for clustering interval data, in D. Banks, L. House, F. McMorris, P. Arabie \& W. Gaul, eds, 'Classification, Clustering, and Data Mining applications', Springer Verlag, pp. 333-339.

Chavent, M., De Carvalho, F. A. T., Lechevallier, Y. \& Verde, R. (2003), 'Trois nouvelles méthodes de classification automatique de données symboliques de type intervalle', Rev. Stat. Appliquées LI(4), 5-29.

Chavent, M. \& Lechevallier, Y. (2002), Dynamical clustering of interval data. optimization of an adequacy criterion based on hausdorff distance, in K. Jajuga, A. Sokolowski \& H.-H. Bock, eds, 'Classification, Clustering, and Data Analysis', Springer Verlag, Berlin, pp. 53-60.

De Carvalho, F. A. T. (1995), 'Histograms in symbolic data analysis.', Annals of Operations Research 55, 289-322.

De Carvalho, F. A. T. \& Souza, R. M. C. (1998), Statistical proximity functions of boolean symbolic objects based on histograms, in A. Rizzi, M. Vichi \& H.-H. Bock, eds, 'Advances in Data Science and Classification', Springer Verlag, pp. 391-396.

De Carvalho, F. A. T., Verde, R. \& Lechevallier, Y. (1999), A dynamical clustering of symbolic objcts based on a context dependent proximity measure., 
in H. e. a. Barcelar, ed., 'Proceedings of the IX International Symposium on Applied Stochastic Models and Data analysis', Universidade de Lisboa, pp. 237-242.

De Souza, R. M. C. R. \& De Carvalho, F. A. T. (2004), 'Clustering of interval data based on city-block distances.', Pattern Recognition Letters 25(3), 353-365.

Diday, E. (1971), 'La méthode des nuées dynamiques', Rev. Stat. Appliquées $\mathbf{1 9}(2)$.

Diday, E. (1988), The symbolic approach in clustering and related methods of data analysis: The basic choices, in H.-H. Bock, ed., 'Classification and related methods of data anlysis', North Holland, Amsterdam, pp. 673-684.

Diday, E. \& Brito, P. (1989), Symbolic cluster analysis, in O. Opitz \& H.-H. Bock, eds, 'Conceptual and Numerical Analysis of Data', Springer Verlag, Berlin, pp. 45-84.

Diday, E. \& Simon, J. C. (1976), Clustering analysis, in K. Fu, ed., 'Digital Pattern Classification', Springer Verlag, pp. 47-94.

Gordon, A. D. (2000), An iteractive relocation algorithm for classifying symbolic data., in W. e. a. Gaul, ed., 'Data Analysis: Scientific Modeling and Practical Application', Springer Verlag, Berlin, pp. 17-23.

Huttenlocher, D. P., Klanderman, G. A. \& Rucklidge, W. J. (1993), 'Comparing images using the hausdorff distance', IEE Transaction on Pattern Analysis and Machine Intelligence 15, 850-863.

Nadler, S. B. J. (1978), Hyperspaces of sets, Marcel Dekker, Inc., New York.

Ralambondrainy, H. (1995), 'A conceptual version of the k-means algorithm.', Pattern Recognition Letters 16, 1147-1157.

Rote, G. (1991), 'Computing the minimum hausdorff distance between two point sets on a line under translation', Information Processing Letters 38, 123-127.

Verde, R., De Carvalho, F. A. T. \& Lechevallier, Y. (2000), A dynamical clustering algorithm for multi-nominal data, in H. A. L. K. et al., ed., 'Data Analysis, Classification and Related methods', Springer Verlag, pp. 387394. 